\title{
Civilisations
}

Revue internationale d'anthropologie et de sciences

humaines

$68 \mid 2019$

Mobilités dans le Sud globalisé : altérité, racialisation et fabrique des identités

\section{Mobilités dans le Sud globalisé : altérités, racialisation et fabrique des identités}

Introduction

Julien Debonneville, Asuncion Fresnoza-Flot et Gwenola Ricordeau

\section{OpenEdition}

Journals

Édition électronique

URL : https://journals.openedition.org/civilisations/5197

DOI : $10.4000 /$ civilisations.5197

ISSN : 2032-0442

Éditeur

Institut de sociologie de l'Université Libre de Bruxelles

Édition imprimée

Date de publication : 1 décembre 2019

Pagination : 11-17

ISBN : 978-2-9602017-3-4

ISSN : 0009-8140

\section{Référence électronique}

Julien Debonneville, Asuncion Fresnoza-Flot et Gwenola Ricordeau, « Mobilités dans le Sud globalisé

altérités, racialisation et fabrique des identités », Civilisations [En ligne], 68 | 2019, mis en ligne le 01 janvier 2021, consulté le 02 janvier 2023. URL : http://journals.openedition.org/civilisations/5197 ;

DOI : https://doi.org/10.4000/civilisations.5197 


\title{
Mobilités dans le Sud globalisé : altérités, racialisation et fabrique des identités - Introduction
}

\author{
Julien DEBONNEVILLE, Asuncion FRESNOZA-FLOT \& Gwenola RICORDEAU \\ Université de Genève ; Université libre de Bruxelles ; California State University, Chico
}

La fabrique de l'altérité et des identités racialisées dans les mobilités transnationales a été essentiellement saisie sous l'angle des migrations Sud-Nord, ou entre "périphéries " et " centres ", mais pas suffisamment dans le cadre des mobilités au sein du Sud globalisé. De nombreux travaux sur la racialisation ont été entrepris à différentes échelles (du local au global) et différentes périodes (Gilroy 1993 ; Balibar \& Wallerstein 1998 ; Grosfoguel 2004), ce qui a permis de pointer la complexité de ces processus de racialisation et d'ethnicisation (Fanon 2001 [1952] ; Barth 1969 ; Poutignat \& Streiff-Fenart 1995 ; Amselle \& M’Bokolo 1999 ; Wimmer 2013), mais aussi leur intersection avec d'autres processus de catégorisation et rapports de pouvoir liés au genre, à la classe sociale et à l'appartenance raciale (Crenshaw 1991 ; Collins \& Andersen 1992 ; Guillaumin 1992 ; Dorlin 2009 ; Falquet et al. 2010). Dans la veine des études critiques de la race (critical race studies), ce dossier propose d'appréhender les multiples circulations transnationales de la fabrique de l'altérité en situations post- ou néocoloniales, en opérant un décentrement du regard à travers la catégorie "Sud globalisé ".

Par "Sud globalisé ", nous faisons référence à un ensemble d'aires géopolitiques contre-hégémoniques et interconnectées entre elles par le biais d'histoires coloniales et/ou de transformations structurelles économiques et sociales. Bien que cette catégorie tende à cristalliser certaines dichotomies, son potentiel heuristique tient avant tout au fait qu'elle visibilise les dynamiques et les processus de reconfiguration qui structurent les rapports de domination à l'échelle mondiale. En l'adoptant, nous mettons ainsi l'accent sur les processus sociaux qui (re)produisent les rapports de pouvoir dans les sociétés contemporaines, mais également les processus de subversion et de décentrement à l'œuvre. En ce sens, ce numéro propose d'interroger les effets performatifs des figures d'altérité et la reconfiguration des identités au regard de la colonialité du pouvoir (Quijano 2007) dans les anciennes colonies.

Pour ce faire, ce numéro est organisé autour de trois axes complémentaires. Le premier, « Mobilités » met la focale sur les migrations mais aussi sur les différentes situations de mobilité dans le Sud globalisé et ce qu'elles impliquent en termes de 
mouvements, de réseaux et de motilités ${ }^{1}$. Le deuxième, "Altérité, altérisation et racialisation ", permet d'analyser les dynamiques de mobilité au regard des multiples formes d'altérisation, et de saisir comment, empiriquement, la racialisation opère au prisme des expériences et des pratiques de mobilité transnationale des actrices et acteurs dans les sociétés de destination et d'origine. Enfin, le troisième axe, "Agentivité et essentialisme stratégique ", à partir de concepts majeurs du champ des études postcoloniales, permet de décrire les stratégies de résistance des actrices et acteurs, mais aussi de questionner les usages sociaux qui sont faits des constructions identitaires.

\section{Mobilités}

La diffusion de la lentille analytique de la « mobilité » - le « tournant de la mobilité » (Urry 2007 ; Adey et al. 2014) en sciences sociales - a permis d'appréhender sous un angle nouveau différentes dynamiques socio-politico-économiques (notamment contemporaines) dans le Sud globalisé. Le concept de "mobilité » revêt des significations variées selon les disciplines et les domaines d'études (Ortar et al. 2018). Une de ses significations les plus fréquentes est celle d' "un changement de condition " en termes de "mouvements, réseaux et motilité " (Canzler et al. 2008 : 2). Le terme " mouvement " réfere ici aux circulations et aux flux ainsi qu'aux trajectoires physiques, sociales, spatiales, géographiques et temporelles de personnes, de groupes des personnes et d'entités " inanimées " telles que des objets, des symboles, des idées ou des émotions (Urry 2007 ; Groes \& Fernandez 2018 ; Salazar 2018). Cette lentille analytique de la mobilité permet de mettre en lumière la circulation et les mécanismes de (re)configuration des formes d'altérisation et des identités racialisées dans des contextes postcoloniaux. Plus précisément, ces différentes formes de mobilité sont façonnées par des réseaux techniques (infrastructures de communication, transports...) et sociaux (relations interpersonnelles institutionnalisées) (Canzler et al. 2008 : 3) qui participent à la circulation de symboles et de représentations sociales altérisantes. Ces réseaux s'apparentent à ce que Duchêne-Lacroix et ses collègues appellent le "régime de spatialité " - " the set of conditions, rules and habits activated in a geographic, social and biographically situated framework which allows, influences or forces the mobility and anchoring practices of specific people for a specific period of time" (2016:275). On peut ajouter à cette définition une dimension historique : par exemple, le passé colonial d'un pays du Sud globalisé et ses liens postcoloniaux avec d'autres sociétés dans le Sud globalisé et dans le Nord globalisé influencent la circulation et la signification des personnes, objets, représentations et idées dans des « espaces sociaux transnationaux " (Faist 1998). Ces circulations produisent et alimentent des imaginaires et des stéréotypes, notamment concernant les appartenances et les identités sur les plans de l'ethnicité, de la nationalité ou encore de la différence racialisée. La mobilité devient dès lors un objet heuristique qui permet de questionner comment les figures d'altérité se coconstruisent en mouvement, à la

1 La notion de "motilité " fait référence à la capacité des personnes, objets, matériaux, symboles, informations, etc. à circuler et à se déplacer dans les espaces géographiques et sociaux, ainsi qu'à travers le temps et les générations (Canzler et al. 2008 ; Kaufmann et al. 2004). 
croisée de différents espaces. En questionnant les différentes territorialités dans lesquelles s'ancre l'altérité, ce dossier interroge ainsi comment les identités circulent et se reconfigurent dans le temps et l'espace à la lueur de différents rapports de pouvoir.

\section{Altérité, altérisation et racialisation}

Dans la veine des approches postcoloniales (Fanon 1952 ; Saïd 1978 ; Mudimbe 1988) et des études subalternes (Guha 1983 ; Spivak 1988 ; Chakrabarty 2000), mais également des études critiques de la race (Bell 1992 ; Crenshaw 1991 ; Delgado 1995) et de l'ethnicité (Barth 1969; Amselle \& M'Bokolo 1999; Wimmer 2013), ce deuxième axe transversal propose de décentrer le regard afin de penser la fabrique de l'altérité et des identités racialisées au sein des mobilités transnationales dans le Sud globalisé. Comme l'a évoqué Homi Bhabha, l'un des auteurs phares de ce qu'on nomme communément aujourd'hui les études postcoloniales, les modes de représentation de l'altérité doivent être questionnés (1994 : 68). Faisant écho à cette recommandation, ce second axe transversal porte ainsi sur le processus de construction de l'altérité dans le Sud globalisé. Il s'agit d'interroger ici les rapports de pouvoir à l'œuvre dans la construction de la différence sur la base d'une pensée dichotomique cristallisant les figures d'un "nous » et d'un " eux ». L'analyse de la production de l'altérité permet de saisir comment l'« autre " est nommé, classé et hiérarchisé. Plus précisément, l'étude de ces processus révèle les mécanismes de catégorisation, de division, de hiérarchisation et d'oppression qui opèrent afin de reléguer des individus ou des groupes sociaux vers une sphère de l'altérité (Delphy 2008). L'altérisation est dès lors comprise comme le résultat de la production de discours, d'imaginaires et de représentations sociales, dont les effets performatifs façonnent les identités et tracent les contours de la différence sexuée, culturelle ou raciale sur la base de marqueurs sociaux et symboliques. Ces derniers délimitent en ce sens les frontières de l'altérité et inscrivent les sujets "indésirables » à la marge du temps et de l'espace. Cette construction de l'altérité est par ailleurs étroitement liée à des dispositifs coloniaux et aux structures de pouvoir qui en ont découlé et opèrent encore aujourd'hui. A ce titre, l'altérisation dans le contexte colonial se caractérise par les formes de racialisation qui en découlent. Il s'agit en ce sens d'un "process by which the empire can define itself against those it colonises, excludes and marginalises. It locates its 'others' by this process in the pursuit of that power within which its own subjectivity is established "(Ashcroft 2004: 158). Appréhender aujourd'hui l'altérisation, en particulier dans un Sud globalisé fortement façonné par la colonisation, ne peut donc se faire sans l'analyse de la "colonialité du pouvoir "(Quijano 2007) afin de prendre en compte comment les différents rapports de pouvoir opèrent et se reconfigurent au rythme des nouvelles mobilités contemporaines (Fresnoza-Flot \& Ricordeau 2017 ; Debonneville 2014).

\section{Agentivité et essentialisme stratégique}

Le concept d'agentivité (agency) est abondamment employé depuis les années 1990 dans les études de genre, dans le sillage des travaux de la philosophe Judith Butler. La définition que celle-ci en donne est large, puisqu'elle désigne " une capacité 
d'agir, de s'émanciper des rapports de domination, passant par l'interruption ou le renversement des normes dominantes " (Butler 2006 : 15). Ses diverses traductions en français, " capacité d'agir, puissance d'agir, agence, agentivité, [...], conscience d'agir » (Haicault 2012), soulignent que, selon les champs des sciences sociales dans lesquels il est utilisé, le terme "agency» a de multiples acceptions.

Néanmoins, ces termes permettent tous d'éclairer la façon dont les individus négocient les normes sociales et subvertissent les identités assignées. A cet égard, l'expression "essentialisme stratégique ", que l'on doit à Gayatri C. Spivak (1988), désigne l'utilisation d'une présupposée " essence " par un individu ou un groupe d'individus à des fins stratégiques. Son utilisation a donné lieu à de nombreuses discussions, notamment dans le champ politique ou elle s'oppose notamment à la formulation d'un " universalisme stratégique » par Paul Gilroy (Robotham 2005). Gayatri C. Spivak, qui ne s'oppose pas à ce qu'elle soit utilisée par d'autres, a néanmoins, depuis, récusé l'expression, en soulignant qu'elle cause des confusions (2011: 78). Comme lentille d'analyse, l'essentialisme stratégique permet de décrire les manières dont les migrants et migrantes développent des pratiques de réappropriation et de (re)valorisation des catégories raciales afin de produire certaines formes d'agentivité. En effet, au cœur de l'expérience de mobilité(s), se trouvent souvent des formes de (re)valorisation et de subversion des catégories raciales, sur des bases nationales ou ethniques.

\section{Présentation des articles}

S'inscrivant dans l'un ou plusieurs des axes exposés ci-dessus, les sept articles rassemblés dans ce dossier s'appuient tous sur de solides recherches empiriques et sont pour la plupart basés sur des enquêtes de terrain de type ethnographique. Les recherches exposées se rattachent à divers champs des sciences sociales (sociologie, anthropologie, histoire, ethnomusicologie) ou peuvent se rattacher aux area studies.

La question de la construction de l'altérité à travers les mobilités par le travail est au centre de plusieurs articles : Noémie Marcus s'intéresse aux Rabidantis, les vendeuses de rue capverdiennes d'origine rurale ; Julien Debonneville, aux travailleuses domestiques originaires des Philippines ; Nassîla Amode, aux travailleurs immigrés haïtiens au Chili. D'autres articles prennent comme point de départ de la construction de l'altérité la circulation d'un objet ou d'une pratique culturelle: Julien Mallet explore les musiques mafana à Madagascar, Joáo De Athayde \& Samuel Lempereur s'intéressent à la pratique de la bourian, une mascarade d'origine brésilienne, par les Agudàs, communauté béninoise d'origine afro-brésilienne.

Les types de mobilité évoquées dans ce numéro sont variées : aux migrations par le travail, évoquées précédemment, s'ajoute la traite esclavagiste. Celle-ci est notamment travaillée par Inès Mrad Dali dans son analyse, en Tunisie, des Noirs tunisiens arrivés des régions subsahariennes en passant par la Libye et qui sont dits d'origine " tripolitaine ». La traite esclavagiste est également au cœur de l'analyse de la pratique de la bourian par Joáo De Athayde et Samuel Lempereur. 
En explorant la construction de l'altérité, les articles réunis dans ce dossier se penchent sur les processus de racialisation. Par exemple, Solenn Al Majali analyse la racialisation des réfugiés subsahariens en Jordanie et Asuncion Fresnoza-Flot étudie la racialisation des enfants de couples belgo-thaïs, appelés luk-kreung (demienfants) en Thaïlande et " métis » en Belgique.

Ces articles décrivent dans le même temps les ressorts variés de l'altérisation : la colonisation et les imaginaires sociaux comme dans le cas des travailleuses domestiques philippines étudiées par Julien Debonneville, ou encore les jeux catégoriels et les répertoires symboliques décrits par Nassîla Amode dans le cas des Haïtiens face au marché du travail chilien. Certains articles mettent la focale sur les stratégies identitaires déployées par les acteurs et actrices. Par exemple, les musiciens de mafana étudiés par Julien Mallet s'inscrivent dans des "Nous" déterritorialisés (blakité globalisée, culture mahoraise...). Dans le cas des enfants de couples belgo-thaïs dans les sociétés belge et thaïlandaise, Asuncion Fresnoza-Flot montre la diversité de leurs stratégies : invisibiliser leurs origines ethniques, accepter et accentuer leurs altérités supposées, et acquérir la nationalité thaillandaise.

Pris dans leur ensemble, ces articles éclairent la fabrique de l'altérité et des identités racialisées dans le contexte des mobilités au sein du Sud globalisé. Ce dossier s'inscrit dès lors dans les débats en sciences sociales sur la globalisation, les mobilités et la fabrique des identités racialisées. Il contribue d'une part au développement d'un champ encore en construction dans l'espace francophone de la recherche, et d'autre part, à la circulation des savoirs et des analyses produits dans d'autres espaces de recherche, notamment anglophones. La richesse des terrains et des analyses réunis dans ce dossier invite par ailleurs à prolonger les réflexions sur la racialisation, la construction de l'altérité et les mobilités, ainsi que les rapports de pouvoir qui structurent les modes de production de la connaissance et les enjeux de décolonisation des savoirs et des terrains (Smith 1999; Mair et al. 2012 ; de Sousa Santos 2014). En effet, analyser la construction de l'altérité fait prendre le risque de reproduire des modes d'altérisation dans la production des savoirs. Il importe dès lors d'interroger la manière dont les privilèges et les formes d'ignorance (Sullivan \& Tuana 2007 ; Cervulle 2012) structurent les modes de production de la connaissance, mais également la façon dont les gestes théoriques tendent à invisibiliser, voire effacer, certaines voix " subalternes ", au point de produire dans certains cas des formes épistémicides (de Sousa Santos 2014). Ce dossier invite dès lors à prolonger les réflexions théoriques sur l'altérité à un niveau plus épistémologique afin d'interroger les rapports de pouvoir, les privilèges et les formes d'invisibilisation qui se jouent dans les gestes théoriques et les pratiques de l'enquête de terrain. 


\section{Références citées}

Adey, Peter, David Bissel, Kevin Hannam, Peter Merriman \& Mimi Sheller (éds), 2014. The Routledge handbook of mobilities. Abingdon, Oxon \& New York : Routledge.

Amselle, Jean-Loup \& Elikia M'bokolo, 1999. Au cœur de l'ethnie: Ethnies, tribalisme et Etat en Afrique. Paris : La Découverte.

Bill Ashcroft, Gareth Griffiths \& Helen Tiffin, 2004. Key concepts in post-colonial studies. Londres : Routledge.

Balibar, Étienne \& Immanuel Wallerstein, 1998. Race, nation, classe. Les identités ambiguës. Paris : La Découverte.

Barth, Fredrik, 1969. Ethnic groups and boundaries: The social organisation of culture difference. Bergen/Oslo \& Londres : Universitetsforlaget/George Allen \& Uwin.

Bell, Derrick, 1992. Faces at the bottom of the well : The permanence of racism. New York: Basic Books.

Bhabha, Homi K., 1994. The location of culture. New York \& Londres : Routledge.

Butler, Judith, 2006. Défaire le genre. Paris : Ed. Amsterdam.

Canzler, Weert, Vincent Kaufmann \& Sven Kesselring, 2008. Tracing mobilities: Towards a cosmopolitan perspective. Burlington, VT \& Farnham, Surrey : Ashgate.

Cervulle, Maxime, 2012. "La conscience dominante. Rapports sociaux de race et subjectivation ", Cahiers du genre 53 (2), pp. 37-54.

Chakrabarty, Dipesh, 2000. Provincialising Europe. Postcolonial thought and historical difference. Princeton : Princeton University Press.

Collins, Patricia Hill \& Margaret L. Andersen, 1992. Race, class, \& gender : An anthology. Belmont, CA : Wadsworth.

Crenshaw, Kimberle, 1991. « Mapping the margins : Intersectionality, identity politics, and violence against women of color », Stanford law review 43 (6), pp. 1241-1299.

de Sousa Santos, Boaventura, 2014. Epistemologies of the South. Justice against epistemicide. Boulder/Londres : Paradigm Publishers.

Debonneville, Julien, 2014. « Les écoles du care aux Philippines. Le devenir travailleuse domestique au prisme de l'Altérité », Revue Tiers Monde 217, pp. 61-78.

Delgado, Richard, 1995. The Rodrigo chronicles : Conversations about America and race. New York: New York University Press.

Delphy, Christine, 2008. Classer, dominer. Qui sont les « autres» ? Paris : La Fabrique Editions.

Dorlin, Elsa, 2009. Sexe, race, classe, pour une épistémologie de la domination. Paris: Presses universitaires de France (PUF).

Duchêne-Lacroix, Cédric, Monika Götzö \& Katrin Sontag, 2016. « The experience of multilocal living. Mobile immobilities or immobile mobilities? ", in Miriam Gutekunst, Andreas Hackl, Sabina Leoncini, Julia Sophia Schwarz \& Irene Götz (éds), Bounded mobilities. Ethnographic perspectives on social hierarchies and global inequalities, pp. 265-281. Bielefeld : Transcript Verlag.

Faist, Thomas, 1998. "Transnational social spaces out of international migration: Evolution, significance and future prospects », European journal of sociology/Archives européennes de sociologie 39 (2), pp. 213-247. 
Falquet, Jules, Helena Hirata, Danièle Kergoat, Brahim Labari, Fatou Sow \& Nicky Le Feuvre (éds), 2010. Le sexe de la mondialisation. Genre, classe, race et nouvelle division du travail. Paris: Presses de Sciences Po.

Fanon, Franz, 2001[1952]. Peau noire, masques blancs. Paris : Le Seuil.

Fresnoza-Flot, Asuncion et Gwenola Ricordeau (éds), 2017. International marriages and marital citizenship : Southeast Asian women on the move. Londres : Routledge.

Guha, Ranajit, 1983. Elementary aspects of peasant insurgency in colonial India. Delhi : Oxford University Press.

Gilroy, Paul, 1993. The black Atlantic: Modernity and double consciousness. Londres : Verso.

Groes, Christian \& Nadine T. Fernandez (éds), 2018. Intimate mobilities. Sexual economies, marriage and migration in a disparate world. Londres : Berghahn.

Grosfoguel, Ramón, 2004. " Race and ethnicity or racialised ethnicities. Identity within global coloniality », Ethnicities 4 (3), pp. 315-306.

Guillaumin, Colette, 1992. Sexe, race et pratique du pouvoir. L'idée de nature. Paris : Côté-femmes.

Haicault, Monique, 2012. "Autour d'agency. Un nouveau paradigme pour les recherches de Genre », Rives méditerranéennes 41, pp. 11-24.

Kaufmann, Vincent, Manfred Max Bergman \& Dominique Joye, 2004. « Motility : Mobility as capital », International journal of urban and regional research 28 (4), pp. 745-756.

Mair, Jonathan, Ann H. Kelly \& Casey High, 2012. «Introduction : Making ignorance an ethnographic object », in Casey High, Ann H. Kelly \& Jonathan Mair (éds), The Anthropology of ignorance : An ethnographic approach, pp. 1-32. Londres : Palgrave MacMillan.

Mudimbe, Valentin-Yves, 1988. The invention of Africa. Gnosis, philosophy, and the order of knowledge. Londres \& Bloomington-Indianapolis : James Currey \& Indiana University Press.

Ortar, Nathalie, Monika Salzbrunn \& Mathis Stock (éds), 2018. Migrations, circulations, mobilités. Nouveaux enjeux épistémologiques et conceptuels à l'épreuve du terrain. Aix-en-Provence : Presses universitaires de Provence.

Poutignat, Philippe \& Jocelyne Streiff-Fenart, 1995. Théories de l'ethnicité. Paris: Presses universitaires de France

Quijano, Aníbal. 2007. « ‘Race’ et colonialité du pouvoir », Mouvements 51 (3), pp. 111-118.

Robotham, Don. 2005. "Cosmopolitanism and planetary humanism : The strategic universalism of Paul Gilroy », South Atlantic quarterly 104 (3), pp. 561-582.

Said, Edward, 1978. Orientalism. New York: Pantheon.

Salazar, Noel B., 2018. Momentous mobilities. Anthropological musings on the meanings of travel. New York: Berghahn.

Smith, Linda Tuhiwai, 1999. Decolonising methodologies. Research and indigenous peoples. Londres \& Dunedin : Zed Books \& University of Otago Press.

Spivak, Gayatri Chakravorty, 1988. "Can the subaltern speak? », in Cary Nelson \& Lawrence Grossberg (éds), Marxism and the interpretation of culture, pp. 271-313. Chicago : University of Illinois Press.

Spivak, Gayatri Chakravorty, 2011. Nationalisme et imagination. Paris : Payot-Rivages.

Sullivan, Shannon \& Nancy Tuana, 2007. Race and epistemologies of ignorance. New York: SUNY Press.

Urry, John, 2007. Mobilities. Cambridge \& Malden : Polity Press. 